\title{
Resolving High Energy Universe Using Strong Gravitational Lensing
}

\author{
Anna Barnacka* \\ Harvard University \\ E-mail: abarnacka@cfa.harvard.edu
}

\begin{abstract}
Extragalactic jets are the largest particle accelerators in the universe, producing radiation ranging from radio wavelengths up to very high-energy gamma rays. Spatial origin of gamma-ray radiation from these sources cannot be fathom due to the poor angular resolution of the detectors. We propose to investigate gravitationally lensed blazars. Cosmic lenses magnify the emission and produce time delays between mirage images. These time delays depend on the position of the emitting regions in the source plane. We combine the precisely measured time delays at gamma rays, well-resolved positions of radio images, a model of the lens and the Hubble constant to elucidate the origin of gamma-ray flares from bright blazar B2 $0218+35$. With this approach, we achieve 1 milliarcsecond spatial resolution of the source at gamma-ray energies. We find that the gamma-ray flares do not originate from the radio core as commonly assumed.
\end{abstract}

35th International Cosmic Ray Conference

10-20 July, 2017

Bexco, Busan, Korea

${ }^{*}$ Speaker. 


\section{Introduction}

Extragalactic jets are the largest particle accelerators in the universe. They transport energy from regions close to supermassive black hole up to hundreds of thousands of light years into intergalactic space. They produce radiation from radio frequencies up to very high energy gamma rays. Despite their broad implication for high energy astrophysics, galaxy formation, and cosmology, the physical nature and internal structure of these jets remain essentially unknown.

Our ability to study high energy emission from these extragalactic jets is severely limited by a poor angular resolution $\left(0^{\circ} .1 \mathrm{deg}\right)$ of gamma-ray instruments: to resolve gamma-ray emission from even the nearest extragalactic jet Messier 87, the angular resolution would have to be improved by a factor of 1000. Most jets are much further away. Spatially imaging any jets will remain impossible for the foreseeable future.

To overcome the technological limitations of gamma-ray instruments, We have proposed to investigate the gravitationally lensed sources harboring extragalactic jets $[4,2]$. Extragalactic jets are gravitationally lensed when a massive galaxy lies along the line of sight between the jet and the observer. The gravitational field of the galaxy deflects light on its way to us and acts as a lens, which both magnifies the emission, and also produces multiple mirage images of the source. Each of the multiple images travels through different lengths and different gravitational potential. The cosmic lens does not change the intrinsic variability of the source but introduces time delays. These time delays depend on the position of the source relative to the cosmic lens, and therefore can be used to study the spatial structure of jets on much smaller scales than are accessible to telescopes.

The Large Area Telescope onboard the Fermi mission (Fermi/LAT [1]) continuously detects gamma-ray radiation from extragalactic jets. Fermi/LAT scans the entire sky every few hours with excellent temporal resolution. This observational strategy provides an ideal set of data for measuring time delays.

We begin by characterizing B2 $0218+35$ in Section 2. We describe results of the lens modeling in Section 3. We describe time delay estimation in Section 4, and introduce the method to resolve the structure of gamma-ray sources in Section 5. We describe the results in Section 6, and conclude in Section 7.

\section{B2 0218+35: Gravitationally Lensed Gamma-ray Blazar}

B2 $0218+35$ is a gravitationally-lensed system with the smallest known Einstein radius (330 mas) $[15,18]$. The system consists of a bright blazar at redshift $z_{S}=0.944 \pm 0.002$ [11], lensed by an apparently isolated spiral galaxy at redshift $z=0.6847$ [9]. The lens bends the emission of the jet into two bright images of the core and extended structures, including an Einstein ring $[17,15,16,18,13,7,8]$.

The first measurement of the time delay using VLA $15 \mathrm{GHz}$ polarization observations yielded a value of $12 \pm 3$ days [12]. Biggs et al. [6] used the results of a three-month VLA monitoring campaign at two frequencies and obtained a time delay of $10.5 \pm 0.4$ days. Cohen et al. [10] used high-precision VLA flux density measurements, over the same epoch as Biggs et al. [6], and measured a time delay of $10.1_{-1.6}^{+1.5}$ days. 


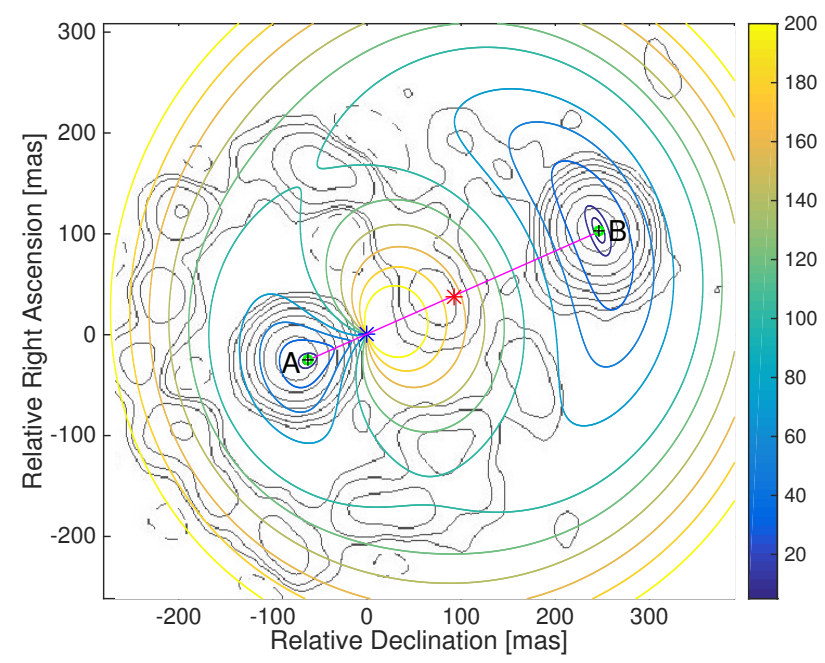

Figure 1: Image plane. The colored contours show the Fermat surface. The coordinates are relative to the reconstructed lens position. Black crosses show the reconstructed mirage image positions. Green open circles show the positions of the $15 \mathrm{GHz}$ mirage images of B2 $0218+35$. The gray contours show the radio emission observed at $1.687 \mathrm{GHz}$. The magenta line indicates the axis connecting the mirage images. The blue star shows the final position of the lens center. The red star indicates the reconstructed position of the $15 \mathrm{GHz}$ radio source.

\section{Lens Modeling Results}

We achieved the best reconstruction for an elliptical singular isothermal sphere [14]:

$$
\psi(r, \theta)=r \theta_{E} \sqrt{1-\varepsilon \cos \left(2\left(\phi-\phi_{0}\right)\right)},
$$

where $\varepsilon$ is an ellipticity of the gravitational potential, $\phi_{0}$ is the position angle of the potential, and $\theta_{E}$ is an Einstein angle defined as:

$$
\theta_{E}=4 \pi \frac{\sigma_{0}^{2}}{c^{2}} \frac{D_{L S}}{D_{O S}},
$$

where $\sigma_{0}$ is the central velocity dispersion of the $3 \mathrm{D}$ velocity field, and $D_{L S}$ and $D_{O S}$ are cosmological distances from the lens to the source, and from the observer to the source, respectively. We also define:

$$
D \equiv \frac{D_{O L} D_{O S}}{D_{L S}}=h d,
$$

where $D_{O L}$ is the distance from the observer to the lens. The parameter $h$ refers to the Hubble constant, $\mathrm{H}_{0}=h \times 100 \mathrm{~km} \mathrm{~s}^{-1} \mathrm{Mpc}^{-1}$.

The fit yields an $\varepsilon \sim 0$, essentially an isotropic SIS. We reconstruct the lens and source positions with an accuracy of 1 mas corresponding to $8 \mathrm{pc}$ in the source plane. The positions of the mirage images are most sensitive to changes of the source position in the tangential direction relative to the images-lens axis. Changing the source position by 1 mas in the tangential direction, moves the mirage images by 5.5 mas. In the radial direction a 1 mas change in the source position 
displaces the image by only 2.7 mas. Table 1 and Figure 1 show that the model reproduces the observed mirage image positions to $0.4-0.8$ mas.

Table 1: Reconstruction

\begin{tabular}{ccc}
\hline Parameter & Value & Difference \\
\hline Image A & $(-0.4,0)$ & 0.4 mas \\
Image B & $(308.6,-128.0)$ & 0.85 mas \\
Time Delay & 10.7 days & $\sim 0.2$ days \\
Magnification Ratio & 3.85 & 0.23 \\
\hline
\end{tabular}

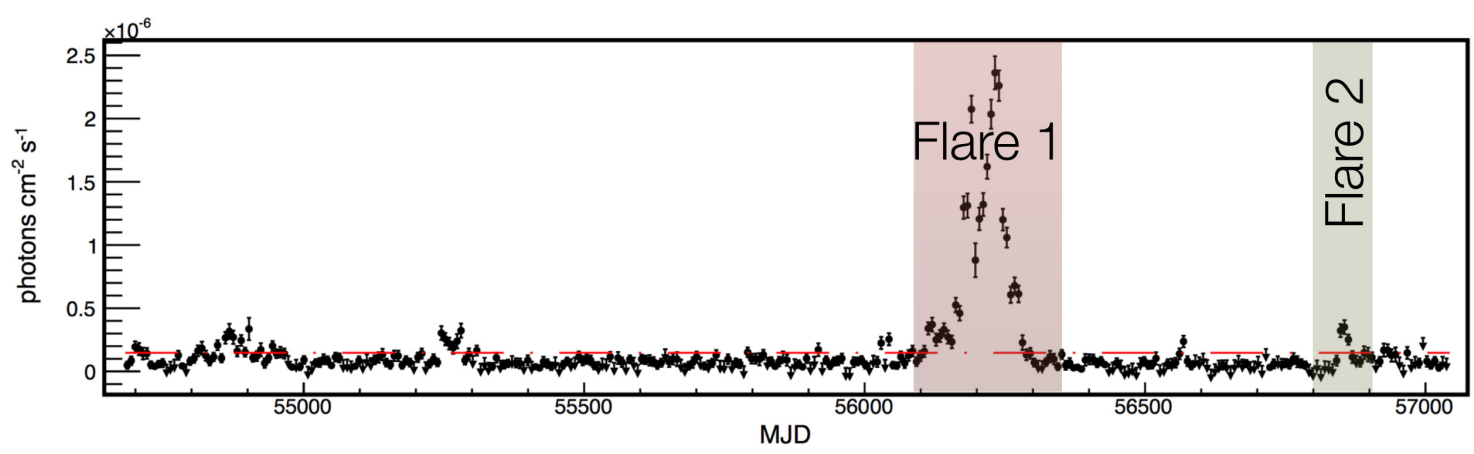

Figure 2: Fermi/LAT light curve of a gravitationally lensed blazar B2 0218+35. Data show time period from August 2008 through February 2015. The fluxes are based on seven-day binning. The energy range is $200 \mathrm{MeV}$ to $300 \mathrm{GeV}$. Time is represented in days since November 17, 1858 (Modified Julian Date: MJD). Figure from [5].

\section{Time Delay Estimation}

On August of 2012 the blazar B2 0218+35 underwent a vigorous outburst of gamma-ray activity. We mark this as "Flare 1" in Figure 2. We analyze this time interval using both the standard Autocorrelation function (ACF) and the Double Power Spectrum (see Figure 3). We obtained a time delay of $11.5 \pm 0.5$ days for the ACF, and $11.38 \pm 0.13$ days using the DPS. The results from ACF and my new DPS analysis are therefore in agreement, however the DPS is more precise by a factor of $\sim 4$. Even more impressive is the statistical significance of the result: this establishes the sensitivity of the method, and also the robustness of its results. We detect gravitationally induced time delay at $>2 \sigma$ level using ACF, and $>4 \sigma$ using DPS. We estimate the significance of the detection using Monte Carlo simulations following [3]. We produce $10^{6}$ artificial light curves of a power law noise. We calculate the chance that a particular time delay signal will appear randomly in the simulated light curve which contains no intrinsic time delays. We display four confidence levels in Figure 3. 

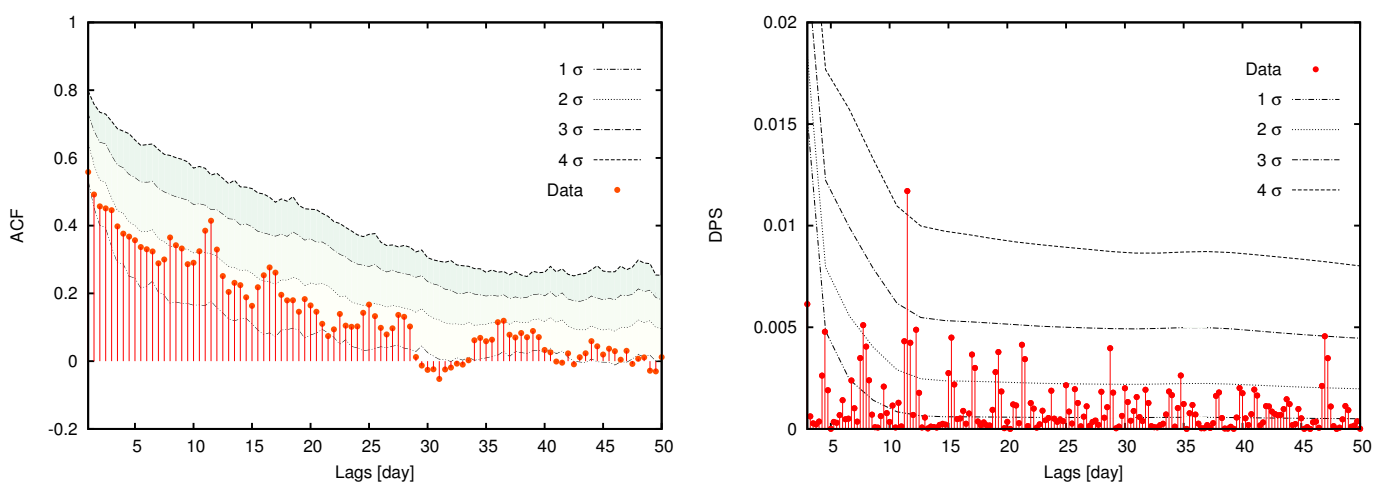

Figure 3: Autocorrelation Function (Left) and Double Power Spectrum (Right) for Flare 1.

\section{The Structure of the Gamma-ray Source}

So far we have used the radio observations and a lens model to reconstruct the origin of the radio core with a resolution of 1 mas (Section 3). The Fermi-LAT observations enable precise determination of the time delay for two gamma-ray flares (Section 4). Here, we locate the sources of gamma-ray emission relative to the radio core by combining the radio source map and the FermiLAT time delays with the well-measured Hubble constant from Planck Collaboration et al. [19].

Barnacka et al. [4] show that the Hubble parameter implied by the time delay is sensitive to any spatial offset between the emission region that produces the resolved mirage images and the site of the variable emission used to measure time delays. Purely on the basis of the physical processes involved, the gamma-ray emission from B2 0218+35 may not be spatially coincident with the radio core [2].

The Hubble parameter, well measured with a variety of independent methods, provides a route to exploring this issue. We can use this precisely measured Hubble parameter to evaluate any offset between the radio core and the site of the variable gamma-ray emission. We call this method the Hubble Parameter Tuning (HPT) approach.

The Hubble parameter enters into the distance ratio in the time delay calculation. For an SIS gravitational potential, the relation reduces to:

$$
h=\frac{d\left(1+z_{L}\right)\left(\theta_{B}^{2}-\theta_{A}^{2}\right)}{2 c \Delta t} .
$$

We have three kinds of constraints on the map of the source from radio to gamma-ray wavelengths: the Hubble parameter, the positions of the lensed images, and the time delay between the images $\Delta t$. If there is an offset between the radio core and the gamma-ray emitting regions, the Hubble parameter derived from the Fermi-LAT time delay will differ from the independently measured "true" value. This difference depends on the distance between the radio core and the spatial location of the flare. The offset in Hubble space corresponds to the spatial offset in the source plane [4].

To locate the origin of the gamma-ray flares from B2 $0218+35$, we first fix $\theta_{A}$ and $\theta_{B}$ to the positions of the resolved images of the $15 \mathrm{GHz}$ radio core. We use these image positions along with the model of the lens and the cosmological parameters to infer the expected time delay for 
the position of the $15 \mathrm{GHz}$ radio core. Table 1 lists the reconstructed position; the value agrees well with the time delay derived from the variability of the radio core although we do not use this delay to compute the Hubble constant. The reconstructed time delay (Table 1) plugged into Equation (5.1) is a consistency check which returns the true value of the Hubble parameter, our reference point.

Next, we calculate time delays for positions within $\sim 10$ mas from the radio core. We use these time delays and positions of the $15 \mathrm{GHz}$ images to compute the Hubble parameter using Equation (5.1). Figure 4 shows these calculated Hubble parameters as a function of the position of the variable emitting region. We call this projection of the model Hubble space.

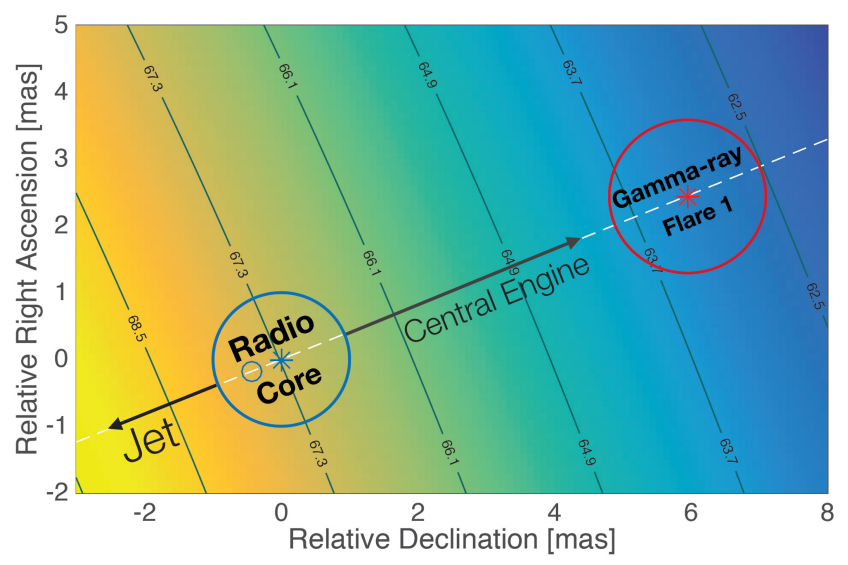

Figure 4: Hubble space. The distances are shown with respect to the position of the radio core (blue circle). The radius of the blue circle corresponds to an uncertainty of 1 mas. The blue star indicates the value of the Hubble parameter based on the reconstructed position of the $15 \mathrm{GHz}$ radio core. The open blue point shows the Hubble parameter derived from the observed positions of the $15 \mathrm{GHz}$ radio images. The dotted line shows the jet projection. Gray arrows show the direction from the radio core toward the central engine and toward the jet. The red circle locates the spatial origin of Flare 1. The radius of the red circle corresponds to the uncertainty in the time delay. The spacing of the white lines in Hubble space corresponds to $1.2 \mathrm{~km}$ $\mathrm{s}^{-1} \mathrm{Mpc}^{-1}$, the $1 \sigma$ uncertainty in the Hubble parameter [19].

\section{The Spatial Origin of Gamma-ray Flare}

Flare 1 has a time delay of $11.33 \pm 0.12$ days. The Hubble parameter obtained based on the position of the $15 \mathrm{GHz}$ radio core and this time delay corresponds to $H_{0}=63.64 \pm 0.67 \mathrm{~km} \mathrm{~s}^{-1} \mathrm{Mpc}^{-1}$. The quoted error corresponds to an error in a time delay of 0.12 days that translates into a spatial resolution of 1.15 mas. Recall that $\mathrm{H}_{0}=67.3 \pm 1.2 \mathrm{~km} \mathrm{~s}^{-1} \mathrm{Mpc}^{-1}$ from [19].

We indicate the position of the $15 \mathrm{GHz}$ radio core in Hubble space in Figure 4. The Hubble parameter estimated for Flare 1 appears as a red dot in Figure 4. The position of Flare 1 in Hubble space is displaced from the radio core. The resolved radio images also constrain the alignment of the jet as indicated in Figure 4 (white dotted line).

The distance between the $15 \mathrm{GHz}$ core and the site of the gamma-ray flare is $6.4 \pm 1.1$ mas displaced toward the central engine. This displacement corresponds to a projected distance of 
$51.2 \pm 8.8 \mathrm{pc}$. The accuracy of the Hubble parameter measured with [19], $\pm 1.2 \mathrm{~km} \mathrm{~s}^{-1} \mathrm{Mpc}^{-1}$, implies that the offset between the resolved radio core and the variable gamma-ray site is significant at the $\sim 3 \sigma$ level.

\section{Conclusions}

By converting temporal resolution into spatial resolution, this measurement of the time delay enables us to model the location of the variable emission. This provides the only constraints of the small scale structure for any extragalactic gamma-ray source. An accuracy in the time delay measurement of 0.13 days corresponds to a spatial position of $\sim 1$ milliarcsecond. The modern gamma-ray instruments resolve the spatial origin of the radiation down to $0.1 \mathrm{deg}$, at best. Therefore, our approach probes jet structure on a scale $>360000$ smaller than limited by spatial imaging with the world's best gamma-ray telescopes.

Comparison of the origin of gamma-ray flare with the radio images resolved down to 0.5 milliarcsecond shows that the gamma-ray flare does not spatially coincide with radio emission, as is commonly assumed. The offset between the radio and gamma-ray emission challenges our understanding how this emission is produced. Moreover, it indicates that attempts to use variable blazers to measure the Hubble constant.

The relativistic jets launched by supermassive black holes are prominent features of high energy astrophysics but are unresolved with current gamma-ray telescopes. As a result, very little is known about their dynamics or internal structure. However, naturally occurring "gravitational lenses" can significantly improve the capability of our telescopes.

The coming era of time domain astronomy will provide well-sampled data for thousands of gravitationally lensed variable sources. The large ensemble of time delays will allow statistical investigation of the origin of emission from extragalactic jets, and will shed new light on our understanding of processes responsible for particle acceleration at large distances from supermassive black holes.

\section{References}

[1] Atwood, W. B., Abdo, A. A., Ackermann, M., Althouse, W., Anderson, B., Axelsson, M., BAldini, L., BAllet, J., BAnd, D. L., BARBiellini, G., AND ET Al. The Large Area Telescope on the Fermi Gamma-Ray Space Telescope Mission. 697 (June 2009), 1071-1102.

[2] Barnacka, A., Geller, M. J., Dell'Antonio, I. P., And Benbow, W. Strong Gravitational Lensing as a Tool to Investigate the Structure of Jets at High Energies. 788 (June 2014), 139.

[3] Barnacka, A., Geller, M. J., Dell' Antonio, I. P., And Benbow, W. Resolving the High-energy Universe with Strong Gravitational Lensing: The Case of PKS 1830-211. 809 (Aug. 2015), 100.

[4] Barnacka, A., Geller, M. J., Dell' Antonio, I. P., And Benbow, W. Strongly Lensed Jets, Time Delays, and the Value of $\mathrm{H}_{0} .799$ (Jan. 2015), 48.

[5] Barnacka, A., Geller, M. J., Dell'Antonio, I. P., And Zitrin, A. The Structure of the Strongly Lensed Gamma-Ray Source B2 0218+35. 821 (Apr. 2016), 58. 
[6] Biggs, A. D., Browne, I. W. A., Helbig, P., Koopmans, L. V. E., Wilkinson, P. N., And Perley, R. A. Time delay for the gravitational lens system B0218+357. 304 (Apr. 1999), 349-358.

[7] Biggs, A. D., Browne, I. W. A., Muxlow, T. W. B., And Wilkinson, P. N. MERLin/VLA imaging of the gravitational lens system B0218+357. 322 (Apr. 2001), 821-826.

[8] Biggs, A. D., Wucknitz, O., Porcas, R. W., Browne, I. W. A., Jackson, N. J., Mao, S., AND Wilkinson, P. N. Global 8.4-GHz VLBI observations of JVAS B0218+357. 338 (Jan. 2003), 599-608.

[9] Browne, I. W. A., Patnaik, A. R., Walsh, D., And Wilkinson, P. N. The Redshift of the Lensing Galaxy in the Gravitationally Lensed System B:0218+35.7. 263 (Aug. 1993), L32.

[10] Cohen, A. S., Hewitt, J. N., Moore, C. B., And HaArsma, D. B. Further Investigation of the Time Delay, Magnification Ratios, and Variability in the Gravitational Lens 0218+357. 545 (Dec. 2000), 578-590.

[11] Cohen, J. G., Lawrence, C. R., And Blandford, R. D. The Redshift of the Lensed Object in the Einstein Ring B0218+357. 583 (Jan. 2003), 67-69.

[12] Corbett, E. A., Browne, I. W. A., Wilkinson, P. N., And Patnaik, A. Radio Measurement of the Time Delay in 0218+357. In Astrophysical Applications of Gravitational Lensing (1996), C. S. Kochanek and J. N. Hewitt, Eds., vol. 173 of IAU Symposium, p. 37.

[13] Jackson, N., Xanthopoulos, E., And Browne, I. W. A. NICMOS images of JVAS/CLASS gravitational lens systems. 311 (Jan. 2000), 389-396.

[14] KNEIB, J. P. Manual of the Lenstool program. Manual of the Lens Tool program (Aug. 2014).

[15] O’Dea, C. P., Baum, S. A., Stanghellini, C., Dey, A., van Breugel, W., Deustua, S., AND SMITH, E. P. Radio and optical observations of 0218+357 - The smallest Einstein ring? 104 (Oct. 1992), 1320-1330.

[16] Patnaik, A. R., Browne, I. W. A., King, L. J., Muxlow, T. W. B., Walsh, D., And WiLKinson, P. N. B0218+35.7 - A gravitationally lensed system with the smallest separation. 261 (Mar. 1993), 435-444.

[17] Patnaik, A. R., Browne, I. W. A., Wilkinson, P. N., And Wrobel, J. M. Interferometer phase calibration sources. I - The region 35-75 deg. 254 (Feb. 1992), 655-676.

[18] Patnaik, A. R., Porcas, R. W., And Browne, I. W. A. VLBA observations of the gravitational lens system B0218+357. 274 (May 1995), L5-L7.

[19] Planck Collaboration, Ade, P. A. R., Aghanim, N., Armitage-Caplan, C., Arnaud, M., Ashdown, M., Atrio-Barandela, F., Aumont, J., Baccigalupi, C., Banday, A. J., AND ET AL. Planck 2013 results. XVI. Cosmological parameters. 571 (Nov. 2014), A16. 